\title{
Las ventajas y desventajas del internet en la sociedad
}

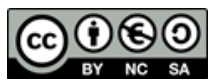

The pros and cons of the internet in society

Efraín Velasteguí López. ${ }^{1}$

Recibido: 16-12-2018 / Revisado: 17-12-2018 /Aceptado: 19-12-2018 / Publicado: 04-01-2019

Resumen.

DOI: $\underline{\text { https://doi.org/10.33262/concienciadigital.v2i1.928 }}$

La finalidad de este trabajo es describir y analizar las ventajas y desventajas del internet en la sociedad, ya que es una red global que influye mucho en la actualidad, este está presente en muchos hogares, instituciones públicas, privadas, centros educativos. El internet también es cuenta como me de comunicación ya que podemos enviar mensajes, hacer llamadas videos llamadas, aparte de esto también nos ayuda con catálogos de biblioteca, hacer compras en aspectos positivos.

Pero también existen aspectos negativos es decir desventajas como el abuso de ciertas redes sociales e incluso el mal uso de aquellas, cabe recalcar que no solo son las redes sociales sino también con ciertos estafadores que se hacen pasar por personas que no son en realidad. El internet, y específicamente hablando de las redes sociales, permiten que nuestra información les sea facilitada a personas que no conocemos pero que sus intenciones son perjudiciales para nuestra integridad y el bienestar de nuestra familia.

Sin lugar a dudas, el internet y las redes sociales revolucionaron la forma de comunicarse, ya que permitió potenciar la simultaneidad e inmediatez de la información, pero, principalmente, por la posibilidad de "subir", sistematizar y clasificar información en un espacio virtual común. En la actualidad no se habla de medios de comunicación, sino de medios de difusión, pasando así de un esquema tradicional a un proceso interactivo, cambiante y dinámico.

Una red social es un espacio creado virtualmente para facilitar la interacción entre personas. Son lugares en Internet donde las personas o usuarios publican y comparten

${ }^{1}$ Ciencia digital, Ambato, Ecuador, luisefrainvelastegui@cienciadigital.org 
todo tipo de información, personal o profesional, con otras personas, conocidos y absolutos desconocidos.

En este informe de investigación vamos a hablar de todos estos aspectos negativos y positivos que de una u otra forma influyen mucho en la vida cotidiana de la sociedad para bien o para mal. El internet facilita la información, como ventaja, pero hay que tener en consideración otros factores que se relacionan casi directamente como las redes sociales como la mal información.

Palabras claves: Internet, sociedad, redes sociales, ventajas y desventajas.

\section{Abstract.}

The purpose of this work is to describe and analyze the advantages and disadvantages of the internet in society, since it is a global network that has a great influence on the present, it is present in many homes, public institutions, private, educational centers. The internet is also counted as me of communication since we can send messages, make calls videos, apart from this also helps us with library catalogs, make purchases in positive aspects.

But there are also negative aspects i.e. disadvantages such as the abuse of certain social networks and even the misuse of those, it should be emphasized that it is not only social networks but also with certain scammers posing as people who are not actually. The internet, and specifically talking about social media, allows our information to be provided to people we don't know but who their intentions are detrimental to our integrity and the well-being of our family.

Undoubtedly, the internet and social networks revolutionized the way they communicate, as it allowed to enhance the concurrency and immediacy of information, but mainly by the possibility of "uploading", systematizing and classifying information in a common virtual space. Today there is no talk of media, but media, moving from a traditional scheme to an interactive, changing and dynamic process.

A social network is a space created virtually to facilitate interaction between people. They are places on the Internet where people or users post and share all kinds of information, personal or professional, with other people, known and absolute unknown. In this research report we will talk about all these negative and positive aspects that in one way or another have a great influence on the daily life of society for better or worse. The internet provides information as an advantage, but other factors that are almost directly related as social networks such as bad information must be taken into account.

Keywords: Internet, society, social networks, pros and cons. 


\section{Introducción.}

Según (Gracia, 2013), (Juncar, 2011), \& (Rojas, 2018), el internet es algo tan habitual como la prensa, televisión, la radio o videos, en casi todos los anuncios aparecen direcciones de internet. En los programas de televisión se puede participar atreves de este medio y un gran número de publicaciones se ocupan de este tema que parece ser la gran revolución en nuestras vidas después de la máquina de vapor y el uso de la electricidad. No hay programa de televisión en que el presentador mencione alguna dirección de internet, mediante en la cual los espectadores pueden encontrar más información. Definir el internet es tan difícil como descubrir el mundo en el que vivimos. Podríamos establecer una comparación entre internet y un mercado: todo el mundo puede ir al mercado de internet, sea como espectador comprador o cliente; ya sea un ciudadano a pie o el príncipe de Gales. En este medio, en principio, no hay diferencias sociales. Coinciden en que el internet se ha convertido en algo indispensable en la vida cotidiana de los seres humanos, ya que nos da la facilidad de muchas cosas por ejemplo trabajar, estudiar desde la casa a través de un computador o Smartphone también hacer compras mediante tiendas online, donde no hay preferencias sociales y pueden ingresas muchas personas, cabe reclamar que el internet está al alcance de todos los hogares ya que existen formas y medios de pago mucho más fáciles, también juega con un papel muy importante en el desarrollo educativo del país, ya que todos los estudiantes al obtener un medio donde puedan adquirir información de manera fácil permite que una nación se vea beneficiada en su desarrollo social, cultural e empresarial. Ellos mencionan que las nuevas TIC están cambiando los comportamientos sociales con una rapidez hasta ahora totalmente desconocida en la historia de la humanidad.

En mi opinión, "el internet puede causar daño como una adicción lo cual no es correcto para una clase social a nivel global, como también nos ayuda también nos perjudica y podemos llegar a un punto donde tenemos q depender de aquello lo cual no es correcto. Podemos llegar a un punto donde la sociedad solo se pasa conectada a través de un computador o Smartphone y así olvidar que lo pueden hacer saliendo de sus hogares. El internet es una de las herramientas que ha marcado tendencia en la actualidad, gracias a todos los beneficios que brinda su aplicación en los hogares. En la actualidad esta tendencia del Internet ha permitido que muchos artefactos electrónicos de uso cotidiano, tales como celulares, tablets, computadoras, etc., puedan comunicarse con la nube y generar reportes."

\section{La sociedad}

Según los autores (Bunge, 2010), (Martínez, 2010) \& (Querol, 2009), la sociedad, la construimos entre todos, en base a los valores, proyectos, compromisos y actuaciones que cada persona pone en juego. Las tecnologías de la información y de la comunicación no determinan cómo debemos usarlas, pero nos obliga a renovar nuestros puntos de vista sobre las realidades a las que nos conducen a las tecnologías, y sobre los futuros que en ellas se proyectan. Internet es la plataforma más económica; sin embargo, ofrece el mejor acceso y 
puede facilitar la creación de un ser humano nuevo, que no esté limitado por ninguna barrera. Es más, no sólo estaremos formando a un ser humano nuevo, sino todo un mundo nuevo, que se materializará dentro de él. Ellos definen a la sociedad como un sistema constituido por cuatro subsistemas, el biológico, económico, político y el cultural. Consideran que en la práctica de la interacción social se combinan los intereses materiales con las emociones y la moral. Atreves de la historia se han estudiado muchos grupos sociales que han ido evolucionando, como las sociedades industriales y las sociedades democráticas, entre otras. Pero la sociedad está regida por deberes y responsabilidades que cada uno debe cumplir, en función de sus capacidades y aptitudes en distintos ámbitos sociales, así tenemos el laboral, educativo, familiar, el de gobierno, etc.

En mi opinión, "la sociedad es más que un conjunto de personas que comparten rasgos, culturas, y otras características. La sociedad es mucho más amplia, generaliza muchos aspectos cotidianos, que compartimos en el entorno laboral, educativo, y natural. Desde la construcción de las primeras sociedades hemos evolucionado de forma impresionante, esta evolución ha traído cambios en la forma de pensar de cada apersona de forma individual y colectiva, con los distintos reglamentos que rigieron durante épocas anteriores. Considero que actualmente la sociedad está conformada, gracias a la evolución social mencionado anteriormente, por tres clases sociales: alta, media y baja, esto ha fomentado actitudes discriminatorias que se intentan apaciguar durante años. Por último, hay que recordar que todos formamos parte de la sociedad desde que somos consiente y participamos activamente en los procesos políticos y laborales que permiten el desarrollo de una sociedad plena, segura, democrática y solidaria."

\section{Las redes sociales}

Según los autores (Canelo, 2011), (Orozco, 2014) \& (Quintero, 2016), las redes sociales son medios que permiten a los usuarios comunicarse entre sí, con los amigos que se encuentren dentro de la misma red, pueden intercambiar fotos, vídeos, mensajes instantáneos, comentarios. Ellos consideran que las redes sociales se pueden utilizar para fines profesionales, temáticas, mixtas, de ocio, personales, entre otros. Las redes que suelen utilizar los jóvenes son las redes personales de ocio, crean encuentros para el crecimiento personal y la profundización de su cultura. Las redes sociales se han convertido en una moda juvenil, están cambiando la realidad social, educativa, económica, política y cultural; aunque la tecnología del Internet no es novedosa, es un medio que ha evolucionado tras el surgimiento de nuevas ideas globales que le han dado mayor fuerza y vigencia. Ellos consideran que las redes sociales son potencian marcas importantes como Coca Cola y McDonalds, mismas que cuentan con páginas en Facebook con millones de fans. Cada vez es más importante para las empresas asegurarse de la exposición de la imagen de marca y su mensaje para atraer clientes y seguidores. Las redes sociales junto con la televisión, radio y el periódico, son parte del ecosistema de las comunicaciones que trabajan juntos para crear una experiencia agradable 
y sin problemas a los consumidores a través de múltiples canales. Del mismo modo, las comunicaciones integradas de marketing están incorporando cada vez más las redes sociales en la mezcla de promoción para llegar a los consumidores en la web y en los dispositivos móviles.

En mi opinión, “considero que el ser humano tiende naturalmente a buscar la compañía de otros semejantes, que piensen y actúen con base en las mismas ideas. Esta tendencia reviste especial importancia para la salud, el ajuste y el bienestar del hombre, lo cual constituye un tipo de apoyo social que se inicia desde el momento en que éste nace y continúa manifestándose durante toda su vida. En consecuencia, es urgente desarrollar un criterio propio para enfrentar "los contras de las redes sociales" ofrecida por el Internet y confrontarla. Así como el internet tiene ventajas y desventajas, de la misma forma y consecuentemente, las redes sociales conllevan beneficio como la rápida información y comunicación de ideas, pensamientos y opiniones, un ejemplo muy claro de esto es el Twitter. Pero, por otra parte, la mente del ser humano es moldeable y vulnerable ante la opresión de estas redes, тиy aparte de ello, esta y otras redes permiten el contacto con personas desconocidas y de malintencionadas."

Tabla N 1. Estudio de usuarios en las redes sociales.

\begin{tabular}{cc}
\hline RED SOCIAL & USUARIOS \\
\hline Twitter & 28 \\
Facebook & 25 \\
Google (Gmail) & 18 \\
Instagram & 16 \\
Pinterest & 11 \\
LinkedIn & 2 \\
Total & $\mathbf{1 0 0}$ \\
\hline
\end{tabular}

Nota: Las redes más utilizadas entre los usuarios.

Fuente: Webempresa20. Blog empresarial, 2018.

En el mundo globalizado en el que vivimos, Internet ya forma parte de nuestras vidas, y así mismo las redes sociales están presentes en todos lados. Sin embargo, hay diversos tipos de redes sociales y cada una con una función específica, porque no todas tienen los mismos objetivos ni tampoco están dirigidos al mismo público, cada una cumple una función específica.

Entre las redes sociales más populares están el Twitter, una red social que permite a sus usuarios compartir a tiempo real en un breve espacio con una extensión limitada de caracteres una idea u opinión sobre temas habitualmente polémicos o del momento. Facebook tiene un objetivo más social, es decir, más de estar en contacto con tus usuarios amigos y establecer 
relaciones sociales. Instagram es una red social cada vez más completa que permite a sus usuarios estar expuestos a contenidos de su interés de forma activa, puesto que permite interactuar entre ellos a través de contenido audiovisual, vídeos en directo, mensajes directos tipo chat o encuestas a tiempo real.

Figura N 1. Representación de las redes sociales más utilizadas por los usuarios.

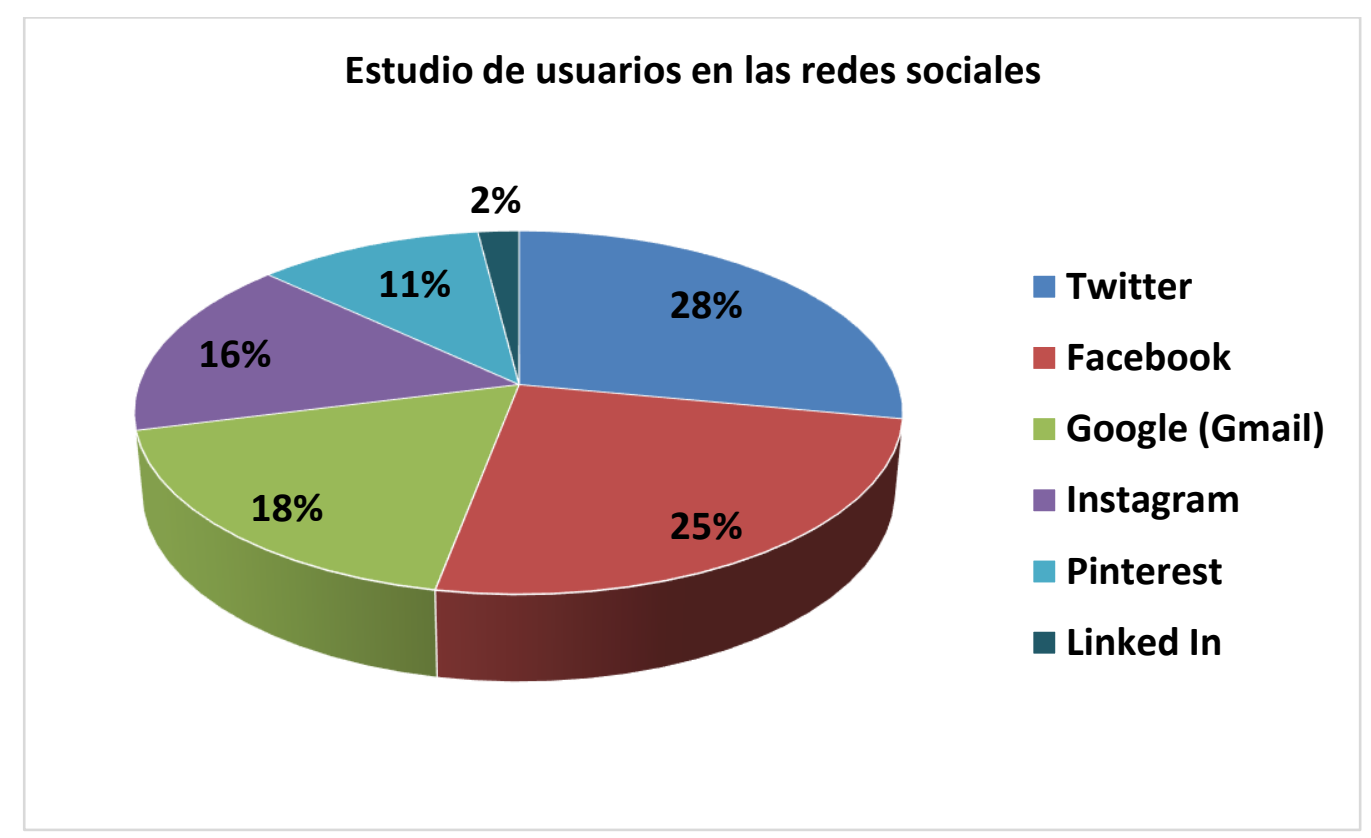

Nota: Porcentajes de uso de cada red social.

Fuente: Webempresa20. Blog empresarial, 2018.

\section{Ventajas del internet}

Según (López, 2015), (Salinas, 2015) \& (Torres, 2013), cualquier avance tecnológico es nuevo en algún momento, consideran entre las ventajas del internet que, la comunicación es más sencilla, acorta distancia a través de la comunicación, ayuda con mucha información, podemos encontrar soportes para cualquier falla de un sistema operativos e intercambiar ideas con otras personas y ayuda con clases virtuales, entre otras. En muy poco tiempo, el internet se ha convertido en una potente herramienta para la comunicación y para la transmisión de información; un potencial del que muchas personas, tanto particulares como entidades empresariales a nivel profesional, y se han aprovechado. La utilización de herramientas de comunicación a través de Internet como las redes sociales, ha revolucionado el mundo de la comunicación debido a sus múltiples ventajas. En la actualidad existe tanta tecnológica que una de ellas es el internet, que nos brinda mucha ayuda ya que tan solo con escribir lo que necesitamos saber y ya tenemos la información y esa es una de las ventajas que tenemos, también nos podemos comunicar a través de el por medio de redes sociales y no solo con personal de nuestro circulo personal, sino que también fuera del como por 
ejemplo amigos, conocidos, familiares que vivan en otra cuidad e incluso país. Cabe recalcar que no todas las personas utilizan el internet la mayoría de casos son los jóvenes para hacer sus tareas o incluso personas en su trabajo. Actualmente nadie cuestiona las ventajas que ofrece el internet como medio de comunicación. La rapidez, la desaparición de la tradicional laminación de tiempo y espacio que dificultaban el trabajo periodístico, la interactividad y el protagonismo de los usuarios en el proceso de comunicación, son los valores añadidos que aporta el internet a los medios de comunicación de masas. Internet también se ha expandido hacia el mundo comercial a través de las empresas virtuales, donde se permite a los usuarios la posibilidad de realizar sus compras o transacciones comerciales, y a las empresas ofertar su catálogo de productos.

En mi opinión, “el internet tiene muchas ventajas, como la facilidad, rapidez, eficacia en la entrega de información, desde el punto positivo, las redes sociales, que están tan presente en la sociedad actual fomentan esto. Pero hay que recordad que existen aspectos negativos en cuanto se trata al internet como medio de comunicación e información. Si bien para los diversos actores sociales las ventajas son muchas, es importante destacar la percepción que tienen los mismos jóvenes usuarios acerca de las desventajas que se pueden encontrar en la red. Considero que en la actualidad la sociedad esta "dominada" por el internet, las nuevas generaciones mantienen mucho más contacto con esta herramienta que en años anteriores, generando consecuencias negativas como un déficit de conocimiento y el apropiado desarrollo de proceso de búsqueda de información. Las ventajas para las nuevas generaciones están en la gran facilidad que tienen los jóvenes para ponerse en contactos con otros, esto permite organizar actividades de su interés como, reuniones familiares, laborales o educativas y a nivel macro, marchas por la defensa de sus derechos con grupos sociales."

Tabla N 2. Ventajas del comercio electrónico vía internet.

\begin{tabular}{|c|c|}
\hline Ventajas para las empresas & Ventajas para los clientes \\
\hline Acceso al mercado mundial. & Interacción. \\
\hline Interacción con los clientes. & $\begin{array}{l}\text { Posibilidad de hacer escuchar su voz y sus } \\
\text { opiniones. }\end{array}$ \\
\hline $\begin{array}{l}\text { Presentación multimedia de la oferta } \\
\text { comercial. }\end{array}$ & $\begin{array}{l}\text { Acceso a una gran oferta en el mercado } \\
\text { (comparación). }\end{array}$ \\
\hline $\begin{array}{l}\text { Reducción de los costos de } \\
\text { comercialización. }\end{array}$ & Control de precios y transparencia. \\
\hline $\begin{array}{l}\text { Estrategias de mercadeo dirigidas a clientes } \\
\text { específicos. } \\
\text { Acceso directo a los clientes potenciales. }\end{array}$ & Comodidad. \\
\hline
\end{tabular}

Nota: Ventajas propuestas a base de la experiencia comercial y la influencia del Internet.

Fuente: Corporación Colombia Digital. Blog digital, 2018. 


\section{Desventajas del internet}

Según (Albornoz, 2007), (Arratia, 2016) \& (David, 2013), gracias al internet es posible conocer e interactuar con muchas personas de todas partes del mundo, pero destacan muchas desventajas sociales y de salud. La búsqueda de información se vuelve mucho más sencilla, sin tener que ir a las bibliotecas tradicionales, pero imposibilita el desarrollo de la lectura como cultura o como parte de la educación, ahora es fácil obtener información en internet, pero muchas veces no es del todo confiable. El acceso a internet que nos brindan los telecentros, con costos de menores a los que ofrecen los ciber cafés. Como bien dicen los autores, el internet es bueno, pero cuando lo utilización con un mal uso es todo lo contrario, muchas veces puede causar adicción en el tema de las redes sociales en muchos casos de los jóvenes, existen los estafadores atreves de internet lo cual hace daño a muchas personas que no lo saben utilizar. Se producen nuevos hábitos y disfunciones en los individuos, formando una clase social a nivel global que podríamos denominar como "los conectados". Esto lleva a un problema detectado entre los usuarios de internet como la adicción a la red. Existen varias desventajas del internet y entre ellas están el aislamiento, la dependencia, vicio de internet, descuido de aspectos personales, etc. Ellos dicen que el internet puede ser fuente de desinformación debido a 1a gran variabilidad en la calidad de los contenidos causa de ansiedad por las diferencias de consejos, testimonios y opiniones o pude cambiar las perspectivas de temas determinados.

En mi opinión, “existen muchos riesgos a los que nos exponemos cuando ingresamos a la red ya sea en búsqueda de información, por diversión o simplemente por curiosidad, los riesgos no son propios de una comunidad o de una edad definida, sabiendo esto podemos ser conscientes de la exposición y las posibles consecuencias que obtendremos si no somos cautelosos en el manejo y apropiado así mismo en la orientación que damos a nuestros educandos o en el mejor de los casos a nuestro mismo núcleo familiar, las posibilidades que ofrece el internet como infraestructura económica y cultural para facilitar muchas de las actividades humanas y contribuir a una mejor satisfacción de nuestras necesidades y a nuestro desarrollo personal, el uso de internet también tiene riesgos. Hay que mencionar que el internet es fácil de manejar, pero de la misma manera debemos tener cuidado con muchas cosas que pasan en la actualidad especialmente con las tarjetas de crédito e incluso en las redes sociales con los secuestros, personas que se hacen pasar por personas que no son. Cabe mencionar que no todo es malo, porque nos brinda de una gran ayuda que si somos responsables la podemos utilizar con frecuencia sabiendo lo que está bien y lo que está mal. El uso del internet puede causar adicción, pudiendo considerar que una persona la padece cuando esta es incapaz de controlar el tiempo que está conectado. Además, el internet puede ser usado para potenciar otras adicciones como juego, compras compulsivas, pornografías, lo cual no es un buen uso que le estamos dando. Entre otras consecuencias están las dificultades de salud, debido al contacto permanente con los medios del internet, teléfonos celulares y computadoras." 


\section{Conclusiones}

- Internet es una de las herramientas que en la actualidad es de las más utilizadas ya sea para comercio, socializar, investigación, educación o simplemente entretenimiento, sin embrago esta herramienta llega a tener sus contras y sus pros, como son las ventajas y desventajas. Una de las desventajas de las redes sociales como el Facebook es que genera miles de secuestros debido a esta gran herramienta sin embargo también se han promovido algunos movimientos como revoluciones pacíficas.

- En la actualidad, si se llega a utilizar de manera incorrecta se puede perjudicar a muchas y tal vez miles de personas, pero de igual manera ayudarlas o darles algún beneficio siempre y cuando se utilice de manera correcta.

- Durante los últimos años, se ha registrado la aparición de redes sociales especializadas en una determinada actividad social o económica, un deporte o una materia, algunos ejemplos son el Facebook, y el twitter que permiten dar a conocer nuestro punto de vista sobre una problemática social. Esto permite satisfacer una necesidad inherente del ser humano de formar parte de grupos con características e intereses comunes.

- Internet es un medio de comunicación al que a diaria no vamos adaptando e integrando tanto a nivel laboral como social y de entretenimiento ya que orece un sin de posibilidades, la comunicación les ha permitido estar más cerca y con mayor rapidez donde se genera la noticia e incluso con los grandes avances que se están dando día con día al ser utilizado por este medio, para un momento dando.

\section{Referencias bibliográficas.}

Albornoz, M. B. (2007). Los usos de Internet: comunicación \& sociedad, Volumen2. QuitoEcuador.

Arratia, O. (2016). Jóvenes.com: internet en los barrios populares de Cochabamba. La Paz, Bolivia: Programa de Investigación Estratégica en Bolivia.

Bunge, M. (2010). Filosofía y sociedad (Primera ed.). México D. F., México: Siglo Veintiuno Editores. 
Canelo, B. F. (2011). Las redes sociales. Lo que hacen sus hijos en Internet. Alicante, España: Editorial Club Universitario.

David, F. R. (2013). Conceptos de administración estratégica (Novena ed.). Naucalpan de Juárez, México: Pearson Educación.

Gracia, R. V. (2013). INTERNET: LA ERA DE LA SOCIEDAD DE LA INFORMACION Y EL CONOCIEMINTO. México D. F., México.

Juncar, J. A. (2011). Easy Internet, 4. Auflage (Lackerbaucer). Madrid, España.

López, R. (2015). La comunicación local por internet. Castellón de la Plana, España: Universitat Jaume I.

Martínez, C. V. (2010). Relaciones con la sociedad. La Coruña, España: Netbiblo, D.L.

Orozco, C. M. (2014). Redes sociales, infancia, familia y comunidad (Pimera ed.). Barranquilla, Colombia: Universidad del Norte.

Querol, R. R. (2009). La sociedad de la información (Primera ed.). (J. Buira, Ed.) Barcelona, España: Universidad Abierta de Cataluña.

Quintero, F. C. (2016). Inseguridad en las redes sociales e Internet: Prioridad en las escuelas de la provincia de Ocaña (Primera ed.). Medellín, Clombia: Instituto Tecnológico Metropolitano.

Rojas, D. H. (2018). Arduino y el Internet de las cosas (Vol. XLV). Alcoy, España: 3Ciencias.

Salinas, S. S. (2015). Internet avanzado: guía profesional. Vigo, España: Ideaspropias Editorial.

Torres, C. (2013). Áreas funcionales para la reflexión: marketing y recursos humanos. Bogotá, Colombia: Editorial Universidad del Rosario. Obtenido de Formared.

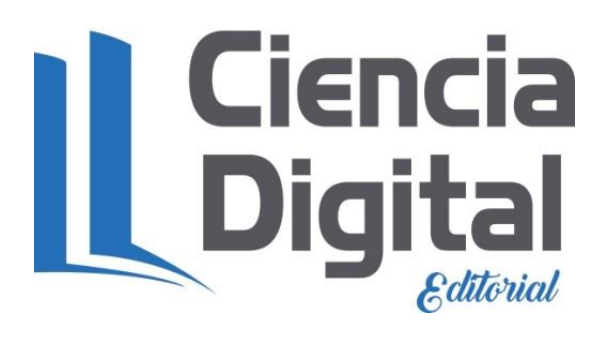


Para citar el artículo indexado.

Velasteguí López, E. (2019). Las ventajas y desventajas del internet en la sociedad. ConcienciaDigital, 2(1), 35-45. https://doi.org/10.33262/concienciadigital.v2i1.928

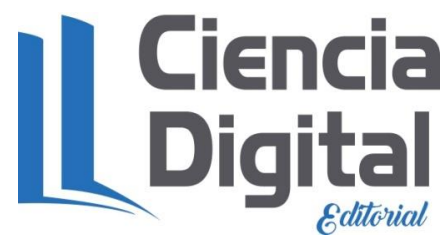

El artículo que se publica es de exclusiva responsabilidad de los autores y no necesariamente reflejan el pensamiento de la Revista Conciencia Digital.

El articulo queda en propiedad de la revista y, por tanto, su publicación parcial y/o total en otro medio tiene que ser autorizado por el director de la Revista Conciencia Digital.

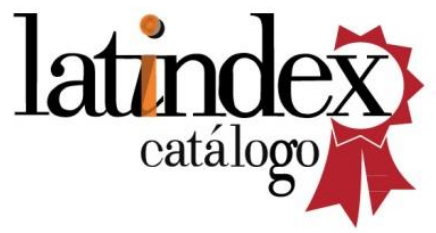

